\title{
Self-Expansion and Compression of Charged Clusters of Stabilized Jellium
}

\author{
ARMANDO VIEIRA, CARLOS FIOLHAIS, AND \\ MARTA BRAJCZEWSKA* \\ Departamento de Fisica, Universidade de Coimbra, P-3000 Coimbra, Portugal; e-mail for M. B.: \\ marta@fteor4.fis.uc.pt
}

JOHN P. PERDEW

Department of Physics and Quantum Theory Group, Tulane University, New Orleans, Louisiana 70118

Received February 25, 1996; accepted February 28, 1996

\begin{abstract}
In a positively charged metallic cluster, surface tension tends to enhance the ionic density with respect to its bulk value, while surface-charge repulsion tends to reduce it. Using the stabilized jellium model, we examine the self-expansion and compression of positively charged clusters of simply metals. Quantal results from the Kohn-Sham equations using the local density approximation are compared with continuous results from the liquid drop model. The positive background is constrained to a spherical shape. Numerical results for the equilibrium radius and the elastic stiffness are presented for singly and doubly positively charged aluminum, sodium, and cesium clusters of 1-20 atoms. Self-expansion occurs for small charged clusters of sodium and cesium, but not of aluminum. The effect of the expansion or compression on the ionization energies is analyzed. For $\mathrm{Al}_{6}$, we also consider net charges greater than $2^{+}$. The results of the stabilized jellium model for self-compression are compared with those of other models, including the SAPS (spherical averaged pseudopotential model). (c) 1996 John Wiley \& Sons, Inc.
\end{abstract}

\section{Introduction}

$\mathbf{T}$ he stabilized jellium model (SJM) or structureless pseudopotential model [1] is a simple modification of the jellium model. In the jel-

*To whom correspondence should be addressed. lium model, the ions are replaced by a continuous charge background of density $\bar{n}=3 /\left(4 \pi r_{s}^{3}\right)$, truncated sharply at a cluster radius $R=r_{s} N_{0}^{1 / 3}$, where $N_{0}$ is the number of valence electrons in the neutral cluster. This model is widely used in the physics of metal clusters, since the precise position of the ions is not important for many physical properties. Stabilized jellium as well as jellium 
may be deformed, but we limit our consideration here to spherical shapes.

Bulk jellium $\left(N_{0} \rightarrow \infty\right)$ is unstable at density parameters which are very different from that of sodium $\left(r_{s}^{B}=3.93 \mathrm{bohr}\right.$ ). (We adopt atomic units in which $e=m=\hbar=1$.) The SJM cures the main deficiencies of the jellium model, namely unrealistic binding energies at all densities, unrealistic bulk moduli for metals with low valence electron density such as cesium ( $r_{s}=5.62$ bohr), and unrealistic surface energies for high valence electron density metals such as aluminum $\left(r_{s}=2.07 \mathrm{bohr}\right)$. The SJM retains the simplicity and universality of jellium, as the only inputs to these models are the bulk density parameter $r_{s}$ and the valence $z$. In the SJM, we subtract the spurious self-repulsion of the jellium charge inside each Wigner-Seitz sphere and then add a constant potential acting on the electrons inside the cluster. This constant potential, different for each metal, is designed so that the bulk metal is stable at the observed valenceelectron density $r_{s}$ (i.e., the pressure $P$ vanishes at the experimental $r_{s}$ ).

In previous works, the surface properties [2], the energetics of small clusters and cohesive properties of bulk metals [3], and a number of fragmentation processes of charged clusters [4] were explored in the framework of the SJM. In all those works the density parameter of the clusters was taken to be $r_{s}^{B}$, the bulk density parameter. However, stabilized jellium permits adjustment of the background density so that the energy per particle of a cluster is minimal $[5,6]$. A neutral cluster with $r_{s}=r_{s}^{*}<r_{s}^{B}$ has a lower energy than a cluster with $r_{s}^{B}$. This effect, which cannot be described in the jellium model, is simply explained, within the liquid drop model (LDM), by the surface tension. It is called "self-compression" and has analogs in nuclear physics [7] and in the physics of helium droplets.

In the present work, the SJM is employed to study not only the self-compression but also the self-expansion of charged clusters of simple metals. The magnitude of self-compression should be reduced for charged systems. Self-expansion is expected if the Coulomb repulsion of the excess charge overwhelms the surface tension. We use the Kohn-Sham equations of density functional theory in the local density approximation (LDA), and the LDM, considering clusters of three different metals $(\mathrm{Al}, \mathrm{Na}$, and $\mathrm{Cs}$ ), which cover the range of physical densities, with single and double positive charges.

Let us see how the main reasoning used to introduce the self-compression of neutral clusters can be extended to the case of charged ones. The constant potential is an average of the difference between an electron-ion pseudopotential and the jellium electron-background potential in a unit cell. It can therefore be expressed in terms of a pseudopotential "core radius" $r_{c}$. Then the bulk stability condition is

$$
\left.\lim _{N_{0} \rightarrow \infty} \frac{\partial}{\partial r_{s}}\left(\frac{E_{0}}{N_{0}}\right)\right|_{r_{s}=r_{s}^{B}}=0,
$$

where $E_{0}=E\left(N_{0}, r_{s}, z, r_{c}\right)$ is the total energy of the neutral cluster in the SJM. Equation (1.1) fixes $r_{c}=r_{c}\left(r_{s}^{B}, z\right)$ for each metal.

The equilibrium positive-background density parameter $r_{s}^{*}$ for a neutral cluster with a finite number $N_{0}$ of valence electrons is the solution of the following equation:

$$
\left.\frac{\partial}{\partial r_{s}}\left(\frac{E_{0}}{N_{0}}\right)\right|_{r_{s}-r_{s}^{*}}=0,
$$

where the derivative is evaluated using the same $r_{c}$ as in Eq. (1.1) (assuming transferability of the pseudopotential from the bulk to the cluster environment).

The elastic stiffness or inverse compressibility of the neutral cluster measures the curvature of the energy with respect to $r_{s}$, around the point $r_{s}^{*}$. It is defined by:

$$
B_{0}=\left.\frac{1}{12 \pi r_{s}^{*}} \frac{\partial^{2}}{\partial r_{s}^{2}}\left(\frac{E_{0}}{N_{0}}\right)\right|_{r_{s}=r_{s}^{*}}
$$

It is a function of $N_{0}, r_{s}^{*}, z$, and $r_{c}$. This second derivative goes over to the bulk modulus $B^{B}$ when $N_{0} \rightarrow \infty$.

If we ionize the cluster, the number of valence electrons becomes $N=N_{0}+\nu$, with $N_{0}$ the number of valence electrons of the neutral system and $\nu$ the number of excess electrons (e.g., for a cluster with a single positive charge, $\nu=-1$ ). The energy of the charged cluster may be evaluated by solving the self-consistent Kohn-Sham [8] equations and filling up in sequence the available single-particle levels. It can also be evaluated using the LDM for charged systems $[9,10]$, in which we disregard 
shell fluctuations. In either case, the equilibrium radius of the positive background is $R=r_{s}^{*} N_{0}^{1 / 3}$, with the equilibrium density parameter defined by

$$
\left.\frac{\partial}{\partial r_{s}}\left(\frac{E}{N}\right)\right|_{r_{s}=r_{s}^{*}}=0
$$

where $E=E\left(N_{0}, r_{s}, z, r_{c}, \nu\right)$ is the total energy of the charged cluster. We have expansion if $r_{s}^{*}>r_{s}^{B}$ or compression if $r_{s}^{*}<r_{s}^{B}$.
The elastic stiffness of the charged cluster,

$$
B=\left.\frac{1}{12 \pi r_{s}^{*}} \frac{\partial^{2}}{\partial r_{s}^{2}}\left(\frac{E}{N}\right)\right|_{r_{s}=r_{s}^{*}},
$$

is different from the stiffness of a neutral cluster. Besides $N_{0}, r_{s}^{*}, z$, and $r_{c}$, it depends on the charge $\nu$.

We shall determine $r_{s}^{*}$ and related physical properties as functions of $N_{0}$ and $\nu$ for different metals (different sets of $r_{s}^{B}$ and $z$ ).

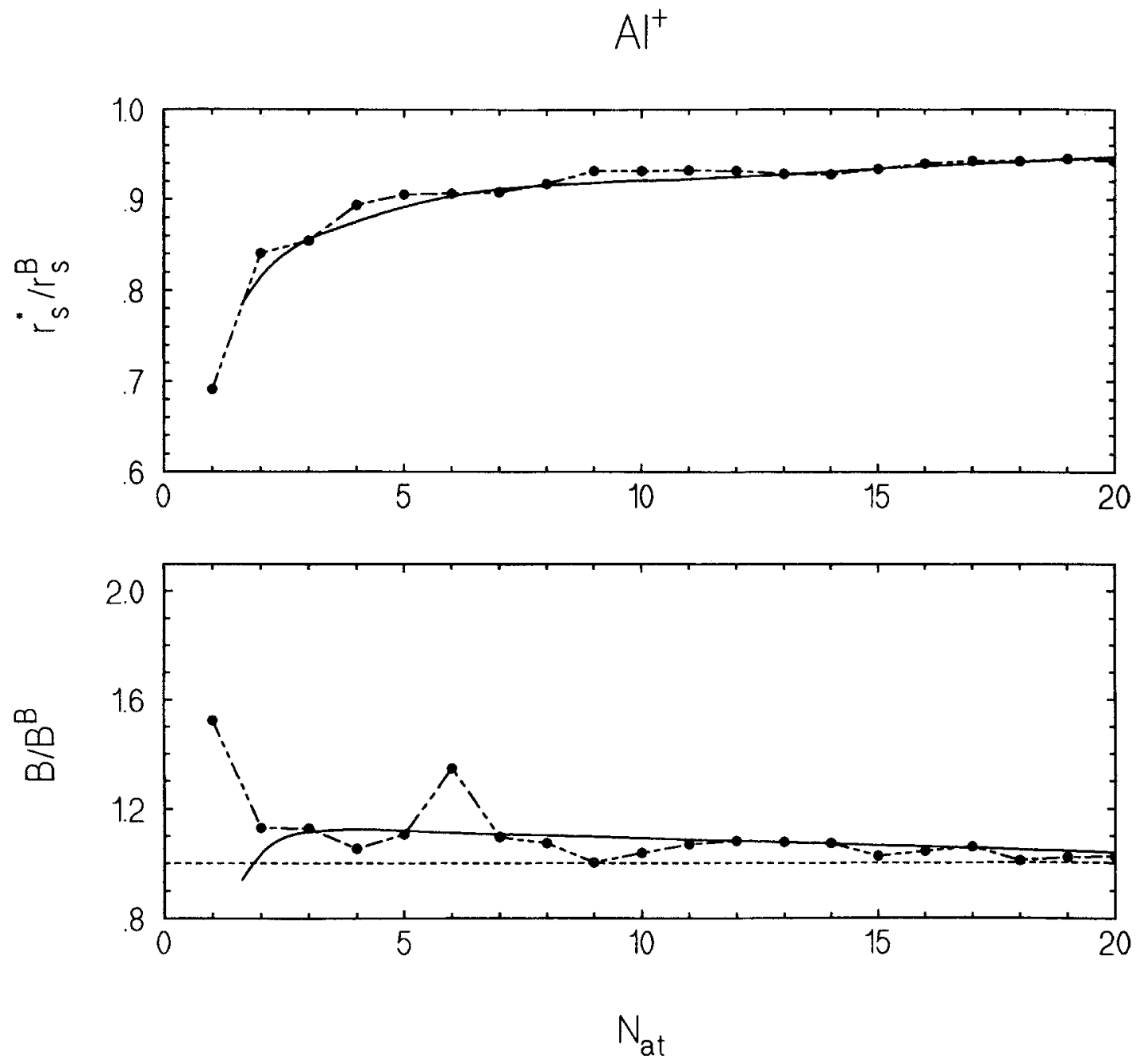

FIGURE 1. (a) Ratio of the equilibrium density parameter $r_{s}^{*}$ to its bulk value $r_{s}^{B}$ as a function of number of atoms $N_{\text {at }}=N_{0} / z$, for a singly charged cluster of stabilized jellium representing $\mathrm{Al}^{+}$. Heavy dots: LDA Kohn-Sham results. Solid curve: Exact numerical solution within the LDM. These results should be compared with those for neutral clusters $[5,6]$. Note, however, that in Ref. [5] the local spin density (LSD) was used instead of the LDA and, in Refs. [5, 6], the densities of $\mathrm{Na}$ and $\mathrm{Cs}$ were $r_{s}^{B}=3.99$ and $r_{s}^{B}=5.63$, in contrast with the present $T=0$ values $r_{s}^{B}=3.93$ and $r_{s}^{B}=5.62$. (b) Ratio of the elastic stiffness to its bulk value as a function of number of atoms for $\mathrm{Al}^{+}$. 
In the next section we elaborate on the LDM for charged clusters. In Section 3, we present and discuss the quantal and liquid drop results, and in Section 4 the main conclusions are drawn.

\section{Energy of the Charged System}

To evaluate the energy of a charged system, we use the Kohn-Sham equations in the LDA, with the Perdew-Wang parametrization [11] for the correlation energy. The quantal equations which were solved are explicitly written in Refs. [3, 4].

The LDM for a charged system deserves more attention, since it is not so well known. In this model, the energy of a neutral cluster is written as a power series of the valence electron number $N_{0}^{1 / 3}$ :

$$
\begin{aligned}
E_{0}=a_{v}\left(r_{s}, z, r_{c}\right) & N_{0}+a_{s}\left(r_{s}, z, r_{c}\right) N_{0}^{2 / 3} \\
& +a_{c}\left(r_{s}, z, r_{c}\right) N_{0}^{1 / 3}+\cdots,
\end{aligned}
$$

where $a_{v}$ is the average energy per electron for a bulk system of uniform density, $a_{s}$ is the surface energy coefficient, and $a_{c}$ is the curvature energy coefficient. This expression can be derived using density functional techniques by developing the density of the cluster around the density profile of the planar surface (Lang-Kohn calculation) [2]. This procedure, which goes under the name of "leptodermous expansion" since it applies to systems with thin skin, allows us to evaluate the surface and curvature energies [7]. Although Eq.

$\mathrm{Al}^{++}$
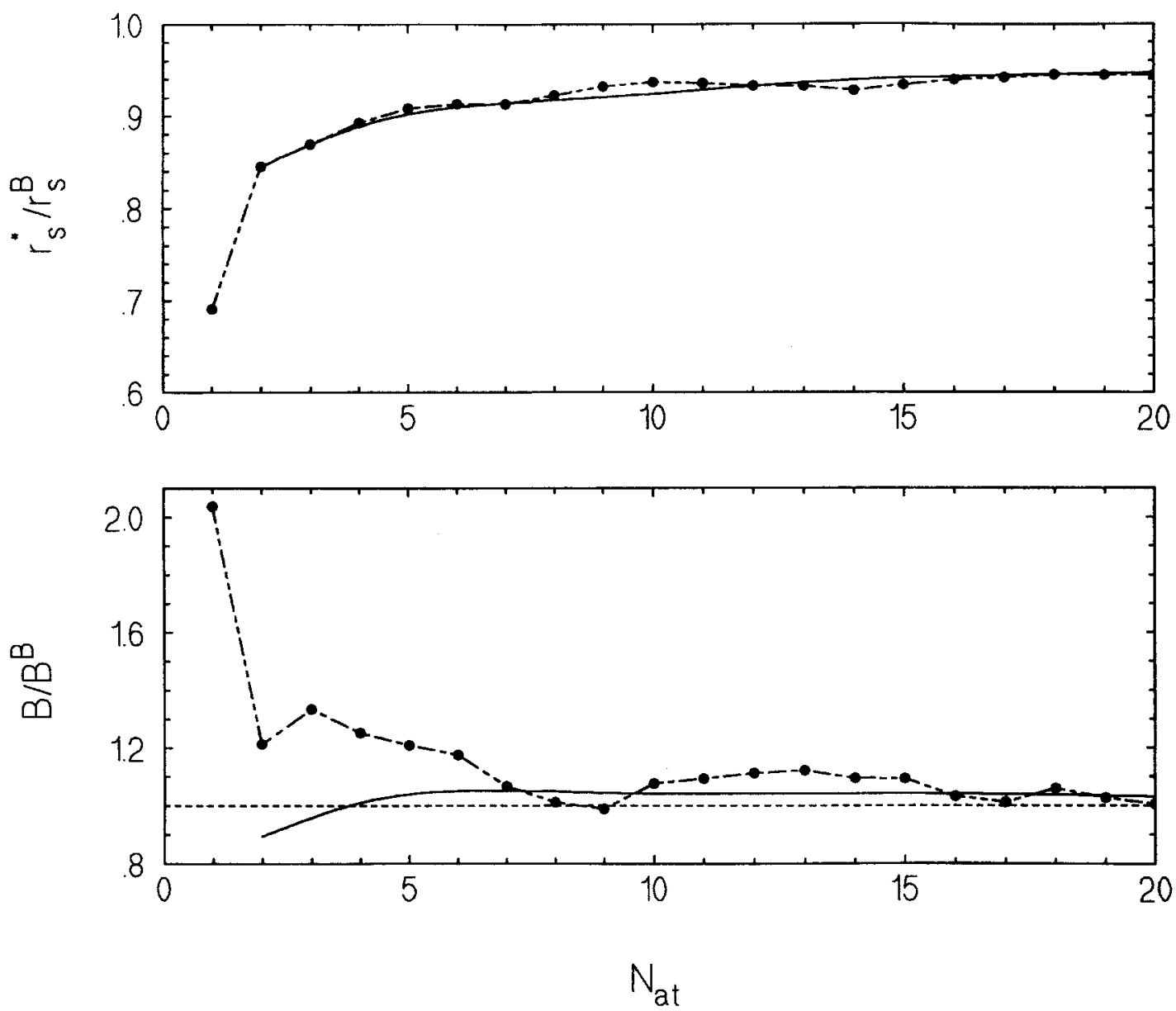

FIGURE 2. Same as Fig. 1, For $\mathrm{Al}^{2+}$. 
(2.1) is strictly valid for $N_{0} \rightarrow \infty$, it provides a useful average of the shell-structure oscillations of the quantal result and may be accurate even in the atomic limit $N_{0}=1[3,12]$.

The energy of a charged cluster may also be described by a continuous approximation. In Refs. $[9,10]$ the following liquid drop formula of a charged cluster with $\nu$ excessive electrons has been obtained in the jellium model:

$$
E=E_{0}-\nu\left(W+\frac{c}{R}\right)+\frac{\nu^{2}}{2(R+\delta)},
$$

where $W$ is the work function, $c$ is a size correction to the work function, and $\delta$ is the distance from the image plane to the jellium edge of the planar surface. In Eq. $(2.2),-(W+c / R)$ is the chemical potential of the neutral cluster; its contribution of order $R^{-2}$ is currently unknown. If we replace the jellium LDM coefficients by those of the SJM, the expression (2.2) is still valid for stabilized jellium, given the similarity of the two energy functionals. It has been used in Ref. [4, 13] without any self-expansion or compression effects. We stress that the LDM formula (2.2) is only justified when the charge $\nu$ is small in comparison with the total number of electrons $N=N_{0}+\nu$.

The ionization energy and the electron affinity may be derived from Eq. (2.2):

$$
\begin{aligned}
I & =E(\nu=-1)-E(\nu=0) \\
& =W+\left(\frac{1}{2}+c\right) \frac{1}{R+\delta}, \\
A & =E(\nu=0)-E(\nu=1) \\
& =W+\left(-\frac{1}{2}+c\right) \frac{1}{R+\delta} .
\end{aligned}
$$

$\mathrm{Na}^{+}$
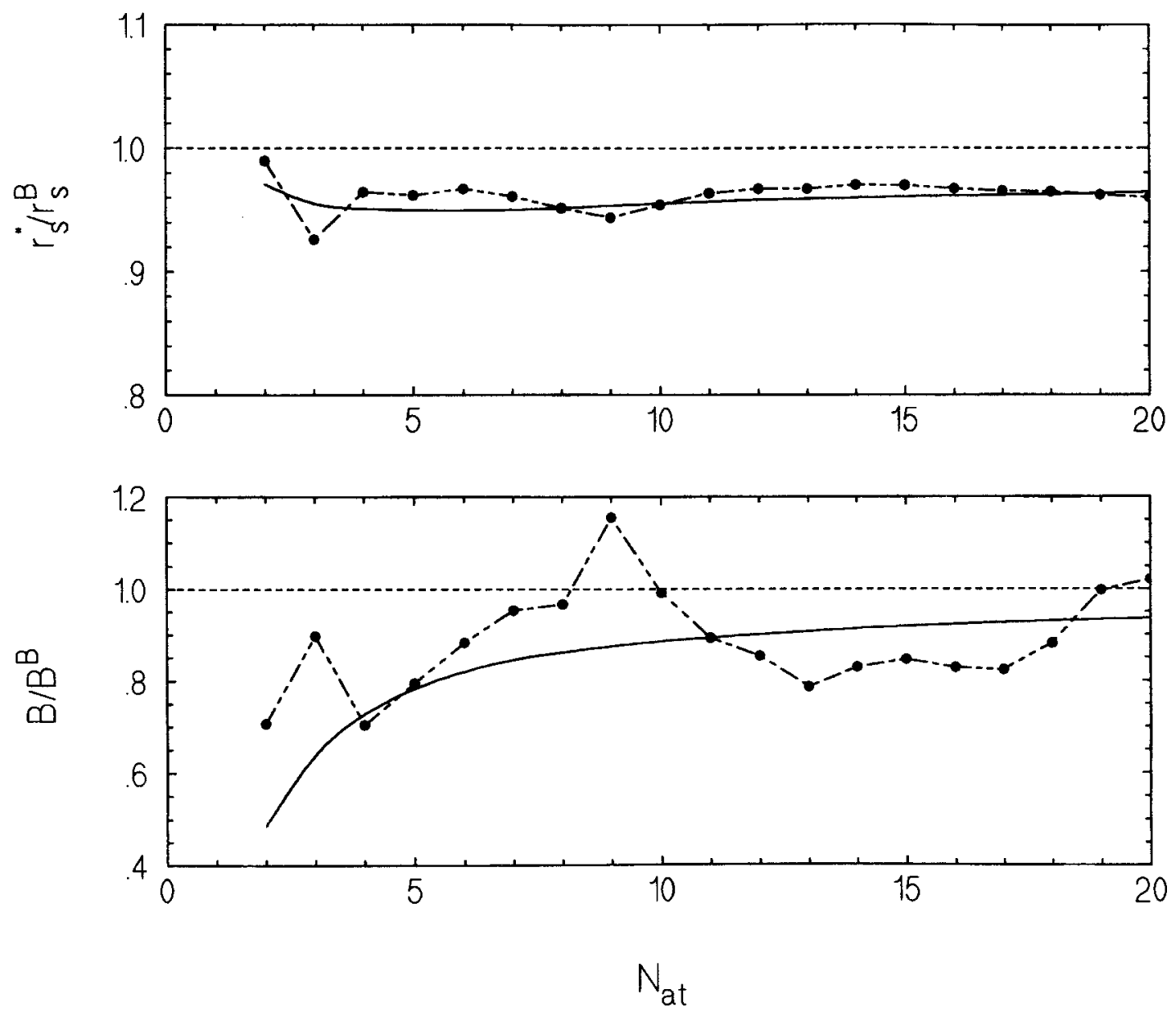

FIGURE 3. Same as Fig. 1 , for $\mathrm{Na}^{+}$. 
Therefore, once values of $W$ and $c$ are available, the SJM can predict ionizations and affinities for finite systems. The work function is given by the same surface calculation which yields $a_{s}$ and $a_{c}$ [2]. Lacking any first principles calculation of $c$, we have evaluated it by taking finite singly charged and neutral clusters and considering an extended Thomas-Fermi approximation (TFDW-4) for the kinetic energy functional of the self-consistent density. Then, fitting the ionization energy by the formula (2.3) yields $c=-0.1$ in the case of $\mathrm{Al}$ [4], close to the value known for jellium $(c=-0.07)$ [14]. Since in the jellium model $c$ is approximately independent of $r_{s}$, we assume that the same is true for the SJM. Finally, $\delta$ was taken from Ref. [15].

The ionization energy is always positive, but within the jellium-LDA approach the electron affinity is negative for small clusters. That is also the case for stabilized jellium-LDA. It is interesting to investigate the number of particles for which the affinity turns our to be positive. From Eq. (2.4), the condition $A \geq 0$ is equivalent to:

$$
N_{0} \geq\left(\frac{\frac{1}{2}-c}{r_{s} W}-\frac{\delta}{r_{2}}\right)^{3} .
$$

More generally, the affinity of order $v$ is defined by

$$
A(\nu)=E(\nu-1)-E(\nu) .
$$

This quantity is positive if

$$
N_{0} \geq\left(\frac{\nu-\frac{1}{2}-c}{r_{s} W}-\frac{\delta}{r_{s}}\right)^{3} .
$$

\section{$\mathrm{Na}^{++}$}
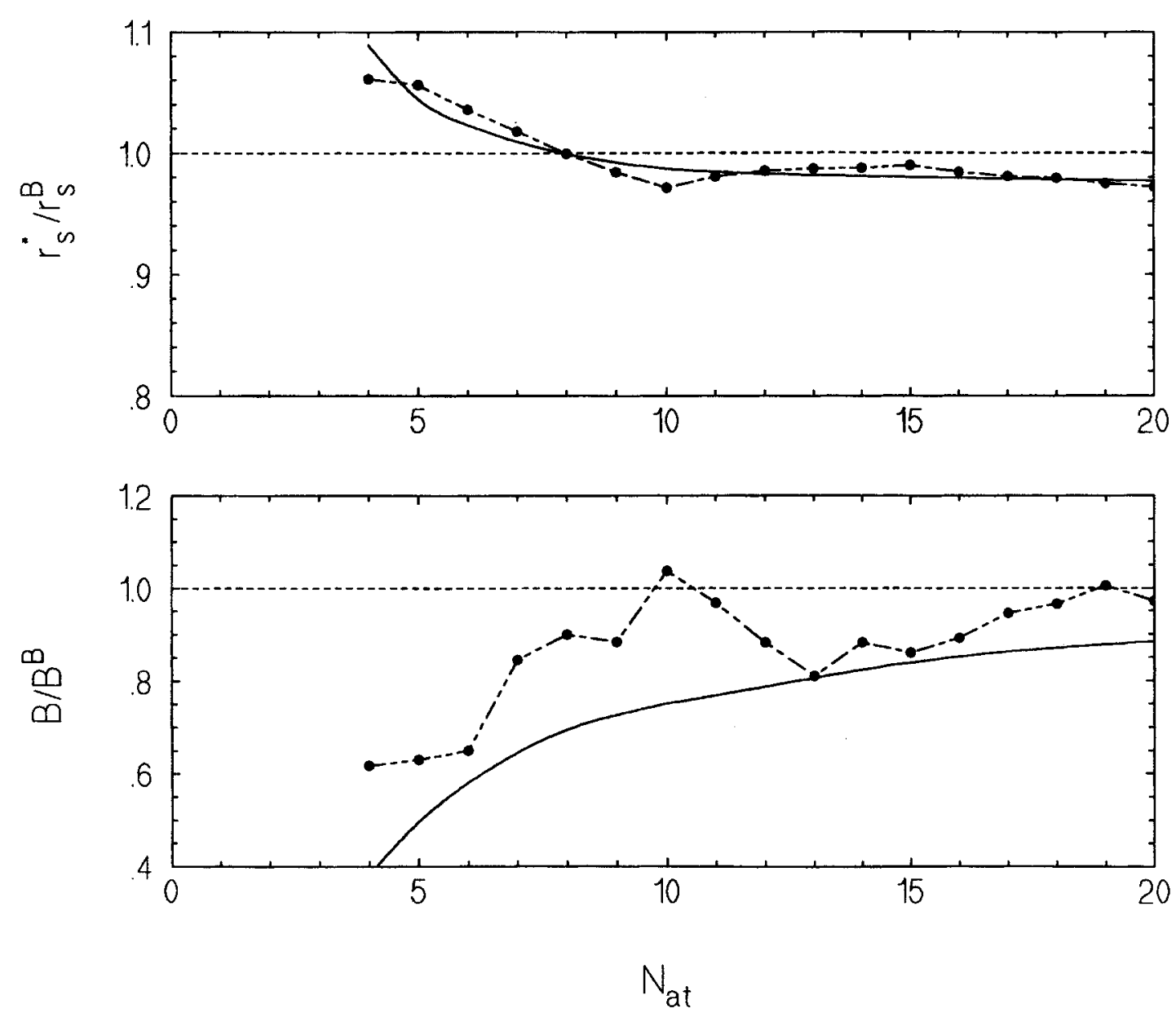

FIGURE 4. Same as Flg. 1 , for $\mathrm{Na}^{2+}$. 
The results on the limits of stability for negative ions are displayed in Table $\mathrm{I}$.

The solution of (1.4) with the energy given by (2.2) is found numerically, since the $r_{s}$ dependence of the LDM coefficients $a_{s}, a_{c}$, and $W$ is known from numerical planar surface calculations. The coefficients $c$ and $\delta$ are assumed to remain constant when compression changes the density of each metal.

The LDM can be used to make simple estimates of the transition from expansion to contraction. We use the Taylor expansion of Ref. [5] around the bulk density parameter:

$$
E=E\left(r_{s}^{B}\right)+\left(r_{s}-r_{s}^{B}\right) E^{\prime}+\frac{1}{2}\left(r_{s}-r_{s}^{B}\right)^{2} E^{\prime \prime}+\cdots
$$

with $E^{\prime}=\partial E /\left.\partial r_{s}\right|_{r_{s}=r_{s}^{B}}=a_{s}^{\prime} N_{0}^{2 / 3}+d_{c}^{\prime} N_{0}^{1 / 3}$, and $E^{\prime \prime}=\partial^{2} E /\left.\partial r_{s}^{2}\right|_{r_{s}=r_{s}^{\mathrm{B}}}$. The equilibrium condition leads to

$$
r_{s}^{*}-R_{s}^{B} \simeq-E^{\prime} / E^{\prime \prime} .
$$

The condition $r_{s}^{*}=r_{s}^{B}$ is then equivalent to $E^{\prime}=0$ or, considering only the surface and the Coulomb term (with $\delta=0$ ):

$$
a_{s}^{\prime} N_{0}^{2 / 3}-\frac{\nu^{2}}{2\left(r_{s}^{B}\right)^{2} N_{0}^{1 / 3}}=0 .
$$

For $\nu=-2$, this happens at $N_{0}=3$ for aluminum, $N_{0}=9$ for sodium, and $N_{0}=11$ for cesium. (The values of the derivatives $\alpha_{s}^{\prime}$ appear in Ref. [5].) Clearly, these guesses for the transition from expansion to compression are only reasonable if the
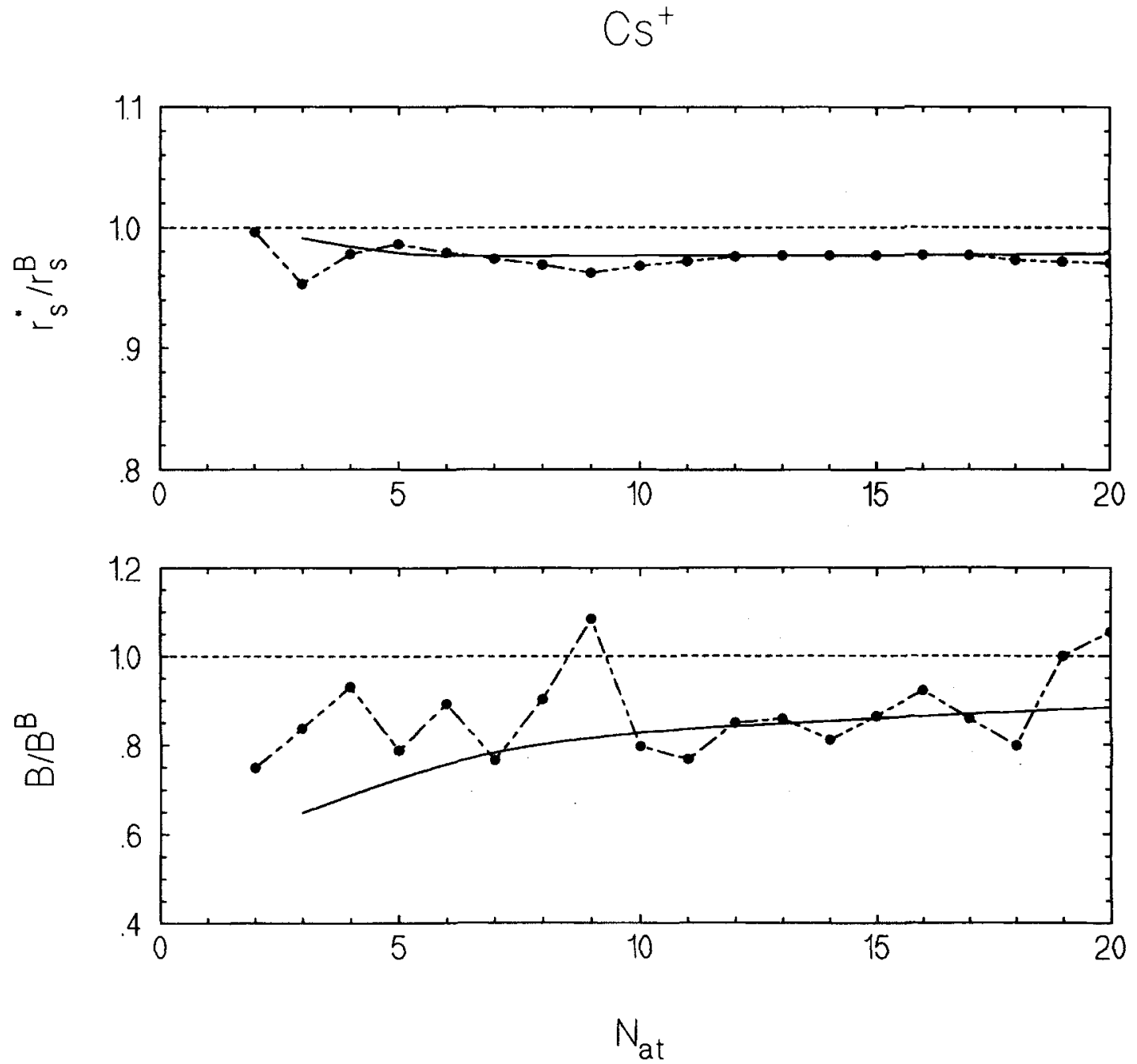

FIGURE 5. Same as Fig. 1 , for $\mathrm{Cs}^{+}$. 

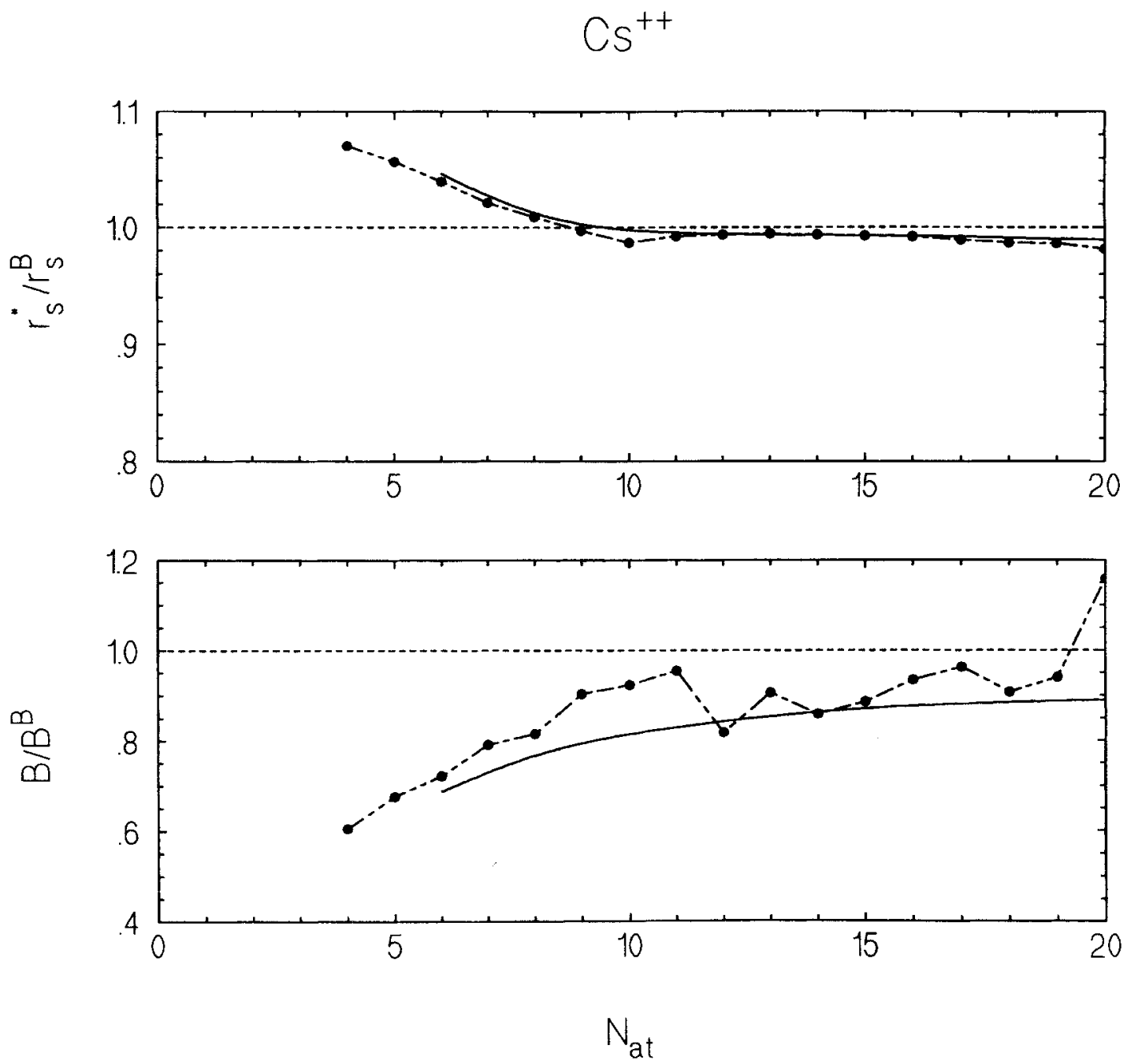

FIGURE 6. Same as Fig. 1, for $\mathrm{Cs}^{2+}$.

TABLE I

The smallest integer value of $N_{0}$ for which the

electron affinity of the $\left(N_{0}+v-1\right)$ valence electron cluster is positive, according to Eq. (2.7). ${ }^{a}$

\begin{tabular}{cccc}
\hline & $\mathrm{Al}$ & $\mathrm{Na}$ & $\mathrm{Cs}$ \\
$\nu$ & $\left(r_{s}^{B}=2.07\right.$, & $\left(r_{s}^{B}=3.93\right.$, & $\left(r_{s}^{B}=5.62\right.$, \\
$z=3)$ & $z=1)$ & $2)$ \\
\hline 1 & 3 & 2 & 2 \\
2 & 90 & 42 & 33 \\
3 & 435 & 210 & 153 \\
4 & 1216 & 554 & 423 \\
\hline
\end{tabular}

${ }^{a}$ The smallest neutral cluster of stabilized jellium that can bind $\nu$ excess electrons has $N_{0}$ valence electrons. The results are similar to those of the jellium model [26]. truncation of the Taylor expansion (2.8) is a good approximation to the exact LDM result, and if the excluded terms in (2.10) may be neglected.

\section{Results}

In Refs. $[5,6]$ we have found for neutral systems with up to 20 atoms that:

1. Small clusters display self-compression $r_{s}^{*} \leq$ $r_{s}^{B}$, i.e., the ionic density is higher in the cluster than in bulk.

2. Quantal shell effects produce local minima in $r_{s}^{*}$ and local maxima in $B$ for closed-shell clusters $N_{0}=2,8$, etc. 
Figures 1(a)-6(a) show the ratio of the equilibrium density parameter $r_{s}^{*}$ to its bulk value $r_{s}^{B}$ as a function of the number of atoms $N_{\mathrm{at}}=N_{0} / z$, for $\mathrm{Al}^{+}, \mathrm{Al}^{2+}, \mathrm{Na}^{+}, \mathrm{Na}^{2+}, \mathrm{Cs}^{+}$, and $\mathrm{Cs}^{2+}$, respectively. From these figures we see that $\mathrm{Al}^{+}$and $\mathrm{Al}^{2+}$ show self-compression for all sizes, although this compression is not so pronounced as in the neutral case. This is due to the high surface tension of Al. On the contrary, $\mathrm{Na}^{2+}$ and $\mathrm{Cs}^{2+}$ exhibit selfexpansion for small clusters and self-compression for large clusters. The transition takes place at $N_{\mathrm{at}}=8$ and $N_{\mathrm{at}}=9$, respectively, using the LDM (and the Kohn-Sham approach gives practically the same transition). $\mathrm{Na}^{+}$and $\mathrm{Cs}^{+}$always show self-compression. The simple rule given by $\mathrm{Eq}$. (2.11) makes a good estimate of the transition from dilatation to contraction, except for $\mathrm{Al}$ where the truncated Taylor expansion is not accurate [6]. In the asymptotic limit $\left(N_{0} \rightarrow \infty\right)$, a fixed net charge is negligible so that the asymptotic expression (Ref. [5]) for $r_{s}$ in the neutral case still holds in the charged case. As in the neutral case, the LDM provides a good average of the quantal results, and quantal shell effects are evident in the curves $r_{s}^{*} / r_{s}^{B}$ versus $N_{\text {at }}$.

The elastic stiffness was calculated numerically by making a least-squares fit of a fourth-order polynomial to the curve $E\left(N_{0}, r_{s}, z, r_{c}, \nu\right)$, around the minimum $r_{s}^{*}$, in the quantal as well as in the liquid drop cases. Figures 1(b)-6(b) show the ratio of the elastic stiffness to its bulk value as a function of the number of atoms, for $\mathrm{Al}^{+}, \mathrm{Al}^{2+}, \mathrm{Na}^{+}$, $\mathrm{Na}^{2+}, \mathrm{Cs}^{+}$, and $\mathrm{Cs}^{2+}$, respectively. We conclude that in the $\mathrm{LDM}$ picture for $\mathrm{Al}^{2+}$, bigger clusters are harder than the bulk and smaller clusters are softer. (The same was found for neutral clusters in Ref. [5], where the asymptotic $N \rightarrow \infty$ correction to the bulk modulus was positive for $\mathrm{Al}$ but negative for $\mathrm{Na}$ and $\mathrm{Cs}$ ). The crossover occurs at $N_{0}=4$. On the contrary, the quantal calculations for $\mathrm{Al}$ always show a cluster stiffness higher than that of the bulk. These observations may have technological implications. $\mathrm{Na}$ and $\mathrm{Cs}$ clusters charged or neutral, are always softer than the bulk in the LDM picture. The quantal results show fluctuations, with some clusters harder than the bulk material.

The role of the charge is to decrease the elastic stiffness. Disagreement between the LDM and the quantal stiffnesses for very small charged clusters indicates that the LDM may be failing in those cases. For some very small clusters, we do not find solutions for the equilibrium radius. The disap- pearance of such solutions is prefigured by a sudden drop in the elastic stiffness.

Ionization energies are affected by the expansion or compression. We can define two ionization energies: one "vertical," where the density parameter is frozen at the value of the neutral system, and another "relaxed," where both the neutral and the charged system are allowed to minimize their energies with respect to $r_{s}$. The first is more realistic, since in a real ionization process the ions do not have time enough to readjust their positions. Figure 7 shows the vertical ionization energy for $\mathrm{Cs}$, in comparison with that calculated for the bulk density (without any compression or expansion). The stronger self-contraction that occurs for closed-shell clusters leads to a stronger binding of the least-bound electron and thus enhances the shell-structure fluctuations of the ionization energy.

\section{Conclusions}

We have examined the self-expansion and selfcompression of singly and doubly positively charged clusters of stabilized jellium. We have found that, for a range of densities which cover $\mathrm{Na}$ and Cs, self-expansion occurs for smaller doubly charged clusters and self-compression for larger ones. However, for high densities, such as that of $\mathrm{Al}$, self-compression prevails for the net charges considered here. If the charge increases further, self-expansion appears even for $\mathrm{Al}$, and for very large charges the phenomenon of "blow up" (Coulomb explosion) arises via disappearance of a solution for the background equilibrium radius (see Figure 8).

Note that the recently proposed "ultimate jellium model" [16], unlike the stabilized or standard jellium models, does not admit solutions for any positively charged clusters.

Typically the charged liquid drop model yields a useful average of the quantal results. But it fails to give results for some very small charged clusters, where the quantal method still applies.

Finally, let us discuss the experimental and theoretical information available on the compression and expansion of charged clusters. Lattice compression or dilatation has been observed in many cases (see Ref. [17]), but the experimental data are contradictory. The observed clusters are bound to 


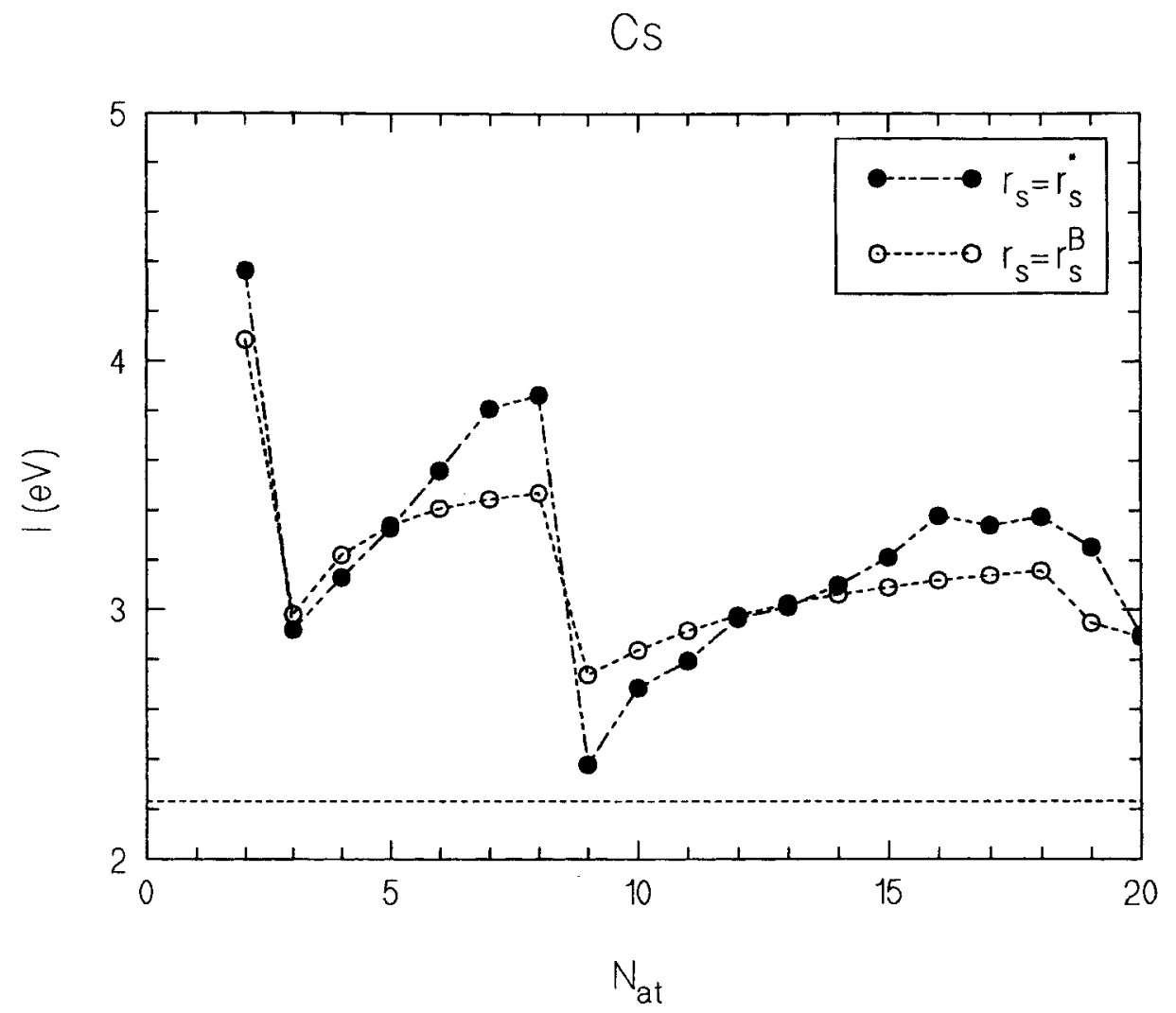

FIGURE 7. First ionization energy of Cs, in the SJM, where the neutral clusters have relaxed background density $r_{s}^{*}$ and the charged clusters have the same density, in comparison with calculations where both neutral and charged clusters have the bulk background density $r_{s}^{B}$. The dashed horizontal line indicates the work function.

a surface, and the effect of the substrate is not well understood.

It is interesting to compare the self-compression of a neutral system predicted by the SJM with a similar compression given by atomistic calculations. The spherical averaged pseudopotential model (SAPS) [18] is a model which has an intermediate degree of sophistication between the SJM and full atomistic calculations. In Refs. $[19,20]$, the SAPS was used to predict an inhomogeneous contraction of a Cs cluster, in which the ions in the inner layers are more closely packed than those near the surface. The SAPS has been used recently to calculate the frequency of the "breathing" mode, where the ions oscillate while maintaining spherical symmetry [21]. In the SJM framework, we can infer the frequency of this mode from the bulk modulus via Eq. (21) of Ref. [21]. The result for $\mathrm{Na}$ $\left(\hbar \omega_{0}=23 N^{-1 / 3} \mathrm{meV}\right)$ does not differ very much from that obtained in the SAPS. (A good fit [21] is given by $\hbar \omega_{0}=a+b N^{-1 / 3}$, with $a=0.6 \mathrm{meV}$ and $b=30 \mathrm{meV}$ ). This suggests that the SAPS is similar to the SJM. The SAPS may also be used to evaluate contraction or dilatation of charged systems and is probably somewhat more realistic than any continuous background model.

In Ref. [22], some of the present authors have evaluated the contraction of six-atom clusters of different metals ( $\mathrm{Li}, \mathrm{Na}, \mathrm{Mg}$, and $\mathrm{Al}$ ), using a linear combination of atomic orbitals local density molecular code but considering only the octahedral shape. The results were only qualitatively comparable with those of the SJM.

Some theoretical calculations on small neutral and charged aluminum clusters with unrestricted geometry are available. In Ref. [23], density functional calculations (with a local spin density approximation for the exchange-correlation energy) and finite-temperature molecular dynamics are applied to neutral and singly charged aluminum clusters, with the number of atoms ranging up to 10. In Ref. [24], an all-electron study on neutral, singly, and doubly charged aluminum clusters (for 2-6 atoms) is carried out within the Kohn-Sham formalism, including gradient corrections to the exchange and correlation energy. In Ref. [25], the 


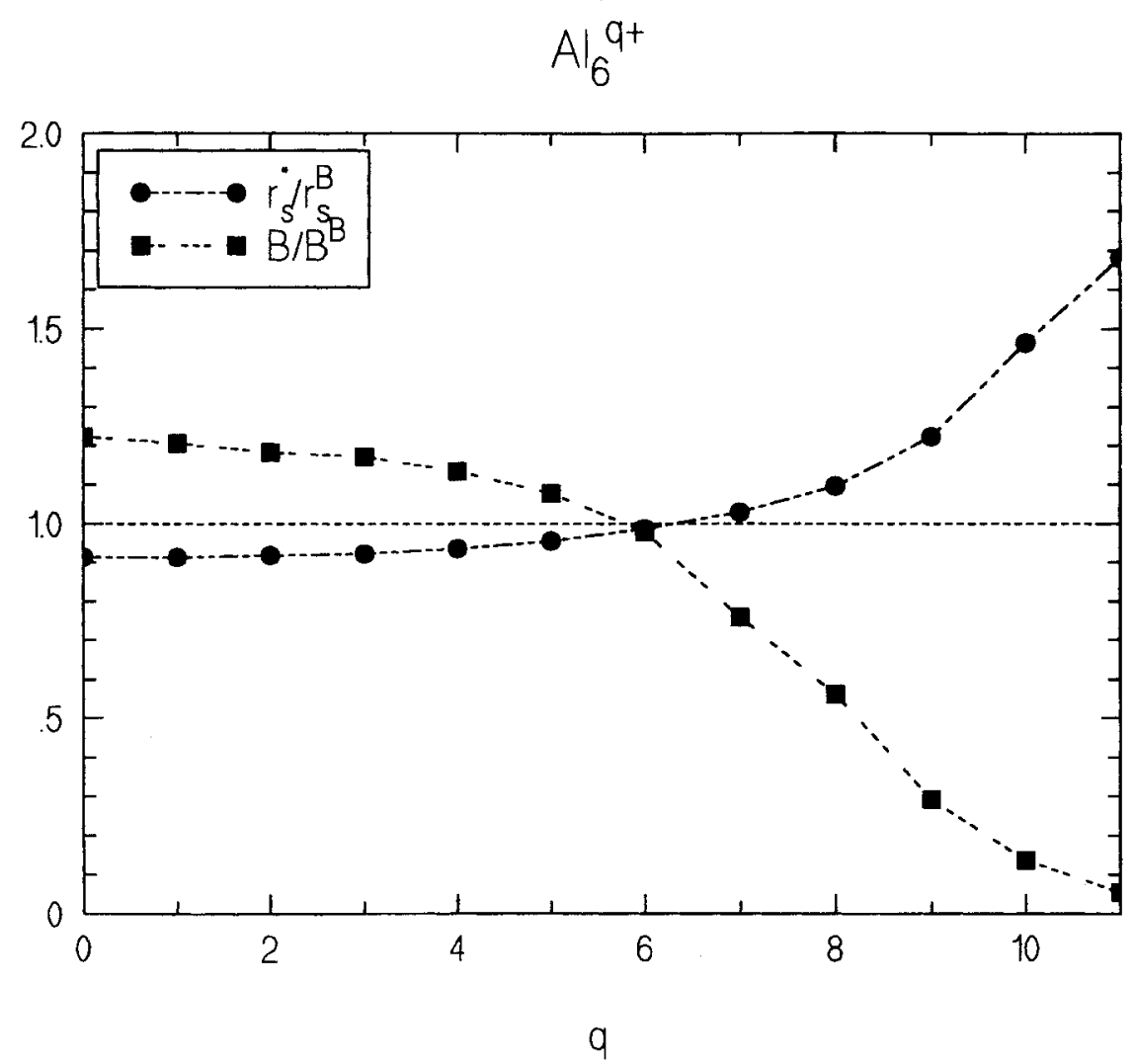

FIGURE 8. Elastic stiffness $B$ and equilibrium density parameter $r_{s}^{*}$ calculated by the Kohn-Sham method for the spherical SJM of $\mathrm{Al}_{6}^{q+}$. There is no self-consistent solution for $q \geq 12$.

structure and properties of singly positively and negatively charged (1-5 atoms) aluminum clusters are investigated by using the linear combination of Gaussian type orbitals method, employing local and nonlocal spin density approximations and a model core potential that allows the explicit treatment of $3 s^{2} 3 p^{1}$ valence electrons. In all these studies the average bond length tends to be smaller in smaller clusters, in agreement with our own results. But, again, the agreement is more qualitative than quantitative. Charged clusters show a smaller bond-length reduction that neutral ones.

We plan to study the SAPS for several charged metallic clusters and to carry out more exact calculations for the charged six-atom clusters with restricted and unrestricted geometries.

\section{ACKNOWLEDGMENTS}

We acknowledge useful discussions with A. Mañanes and L. C. Balbás. The work of two of the authors (J. P. P. and C. F.) was supported by
NATO Collaborative Research Grant No. 910623. This work has been partially supported by the Praxis XXI Program (Project 2/2.1/FIS/26/94), the Commission of the European Communities (Contract ERB-SC1-CT92-0770), the Junta Nacional de Investigação Científica e Tecnológica (Project PBIC /C /FIS/2216/95), and the U.S. National Science Foundation (Grant No. DMR95-21353).

\section{References}

1. J. P. Perdew, H. Q. Tran, and E. D. Smith, Phys. Rev. B 42, 11627 (1990).

2. C. Fiolhais and J. P. Perdew, Phys. Rev. B 45, 6207 (1992).

3. M. Brajczewska, C. Fiolhais, and J. P. Perdew, Int. J. Quantum Chem. S 27, 249 (1993).

4. A. Vieira, M. Brajczewska, and C. Fiolhais, Int. J. Quantum Chem. 56, 239 (1995).

5. J. P. Perdew, M. Brajczewska, and C. Fiolhais, Solid State Commun. 88, 795 (1993).

6. M. Brajczewska, C. Fiolhais, A. Vieira, and J. P. Perdew, in Many-Body Physics, C. Fiolhais et al., Eds. (World Scientific, Singapore, 1994), p. 455. 
7. M. Brack, C. Guet, and H.-B. Håkansson, Phys. Rep. 123, 275 (1985).

8. W. Kohn and L. J. Sham, Phys. Rev. 140, A1133 (1965).

9. J. P. Perdew, in Condensed Matter Theories, J. Keller, Ed. (Plenum, New York, 1989), vol. 4.

10. M. Seidl and M. Brack, Annals of Physics (N.Y.) 245, 275 (1996); M. Seidl and J. P. Perdew, Phys. Rev. B 50, 5744 (1994).

11. J. P. Perdew and Y. Wang, Phys. Rev. B 45, 13244 (1992).

12. J. P. Perdew, P. Ziesche, and C. Fiolhais, Phys. Rev. B 47, 16460 (1993); P. Ziesche, J. P. Perdew, and C. Fiolhais, Phys. Rev. B 49, 7916 (1994). These articles describe voids and monovacancies within the stabilized jellium and liquid drop models.

13. A. Vieira and C. Fiolhais, Z. Phys. D, to appear.

14. E. Engel and J. P. Perdew, Phys. Rev. B 43, 1331 (1991).

15. A. Kiejna, Phys. Rev. B 47, 7361 (1993).
16. M. Koskinen, P. O. Lipas, and M. Manninen, Z. Phys. D 35, 285 (1995).

17. E. L. Nagaev, Sov. Phys. Usp. 35, 747 (1992).

18. M. P. Iñiguez, M. J. López, J. A. Alonso, and J. M. Soler, Z. Phys. D 13, 171 (1989).

19. A. Mañanes, J. A. Alonso, U. Lammers, and G. Borstel, Phys. Rev. B 44, 7273 (1991).

20. A. Mañanes, private communication.

21. J. G. Aguilar, A. Mañanes, F. Duque, M. J. López, M. P. Iñiguez, and J. A. Alonso, Int. J. Quantum Chem., to appear.

22. F. Nogueira, C. Fiolhais, J. He, J. P. Perdew, and A. Rubio, J. Phys.: Condens. Matter 8, 287 (1996).

23. R. O. Jones, Phys. Rev. Lett. 67, 224 (1991).

24. A. Martínez and A. Vela, Phys. Rev. B 49, 17464 (1994).

25. P. Calaminici, N. Russo, and M. Toscano, Z. Phys. D 33, 281 (1995).

26. J. P. Perdew, Phys. Rev. B 37, 6175 (1988). 